\title{
Unilateral rhinolith in an adult man: a case report
}

Yusof ANM ${ }^{1}$, Salahuddin $Z^{2}$

\begin{abstract}
Foreign bodies in the nose are rarely seen among adults. Rhinoliths are uncommon intranasal foreign bodies that have a very low incidence. They are often asymptomatic may be up to many years and subsequently present with nasal symptoms. We reported a case of an adult man with unilateral rhinolith, who complained of unilateral nasal blockage and foul-smelling nasal discharge. He was diagnosed with rhinolith endoscopically and successfully removed under general anaesthesia.
\end{abstract}

Keywords: Rhinolith; foreign body; adult

Bangladesh Journal of Medical Science Vol. 16 No. 03 July'17. Page : 448-451

\section{Introduction}

Rhinoliths are calcareous material (stone like) in nasal cavity that formed from calcified nidus. They can be either from exogenous and endogenous origin. The exogenous source is foreign bodies placed in nasal cavity while the endogenous is from body part. ${ }^{1}$ Even though nasal foreign bodies are frequently seen in children, the incidence of rhinolith foreign body is only $0.01 \%$ in Otorhinolaryngology (ORL) clinic. ${ }^{2}$ They can be presented in all ages but apparently rhinoliths are more common among adult female. ${ }^{3,}$ ${ }^{4}$ In adult, they are mostly due to injury from road traffic accident, trauma or coexisting mental illness. ${ }^{5}$ Initially, rhinoliths are asymptomatic which often accidentally detected from dental radiograph during dental checkup. ${ }^{3}$ As they gradually grow bigger and significantly enlarged, they may manifest symptoms of unilateral nasal blockage, foul smelling nasal discharge, epistaxis, nasal swelling, oral malodour or nasal pain. ${ }^{3}$ Left untreated rhinoliths can cause complications such as sinusitis, septal perforation, oro-antral fistula and deviated nasal septum. ${ }^{3}$

\section{Case report}

A 49-year-old man was referred to the ENT clinic with chief complaint of persistent right nasal blockage and unilateral foul-smelling nasal discharge for 4 months duration. There were also symptoms of rhinorrhea and anosmia. However, he denied facial pain, epistaxis and constitutional symptoms. On further questioning, there was no history of trauma or nasal operation. Moreover, he did not recall any history of foreign body in the nose previously.

Anterior rhinoscopy examination noted mild bilateral inferior turbinate hypertrophy with foul smell odour. Cold spatula test showed reduce airflow from the right nostril. Oropharyngeal examination was normal. There was no neck node palpable. Then, proceed with rigid nasoendoscopic examination revealed stone like mass with minimal whitish discharge occupied at the right floor of the nasal cavity. It was dark brownish, irregular hard mass on probing. These findings were consistent with the right rhinolith.

Removal of rhinolith attempted but failed as the patient complained of pain. He was then undergone examination under anaesthesia and removal of foreign body under general anaesthesia. The rhinolith was successfully removed endoscopically by crushing it into fragments with forceps. It was located at inferior meatus in middle part of nasal cavity. There was no septal perforation, oroantral perforation, nasal polyp, septal deviation, granulation tissue or pus discharge. The osteomeatal complex looked normal. There was no massive bleeding during the procedure and he did not require anterior nasal pack. He was well post operatively without any complication.

1. Asfa Najmi Mohd Yusof, Department of Otorhinolaryngology-Head \& Neck Surgery, School of Medical Sciences, UniversitiSains Malaysia, 16150 Kota Bharu, Kelantan

2. Zulkiflee Salahuddin, Department of Otorhinolaryngology-Head \& Neck Surgery, Hospital Raja PerempuanZainab II, Kota Bharu, Kelantan, Malaysia

Correspondence to: Asfa Najmi Mohd Yusof, Department of Otorhinolaryngology-Head \& Neck Surgery, School of Medical Sciences, UniversitiSains Malaysia, 16150 Kota Bharu, Kelantan

Email: bluekitten4477@gmail.com 


\section{Discussion}

Rhinolith term is derived from Greek, "Rhino" means nose and "lith" means stone. Rhinolith or rhinolithiasis is defined as a partial or total calcified mass in the nasal cavity which forms around a foreign body nidus. ${ }^{7}$ It was first discovered in 1654 by Bartholin. ${ }^{1}$ The rhinolith cases are rarely reported with an estimation of 1 in 10000 patients attended at ORL clinic ${ }^{2}$ and some literature reported 1 new case per year. ${ }^{8}$

Rhinoliths can be classified into 2 types which are endogenous and exogenous. ${ }^{6}$ The endogenous type is when the rhinolith formed around normal body material such as blood clot, bone fragment, dried pus or tooth. ${ }^{7,9}$ While the exogenous type is formed around the foreign body that inserted into nasal cavity such as fruit seed, sand, wood, peas, beads, button or gauze tampon. ${ }^{6}$ The exogenous source is more frequently reported comparing to endogenous type. ${ }^{9}$ These might be due to foreign body enter the nose during trauma that unaware by the patient. Also neglected and forgotten foreign body into the nostril during childhood period. ${ }^{10}$ Similarly in our case, there was yellowish material at central core of rhinolith identified after crushing the rhinolith. It was unlikely exogenous material but it might be the aftermath of endogenous origin such as dried pus that induce the rhinolith formation around it.

The exact pathogenesis is not well understood. It is believed that the rhinoliths are produced by layers of mucin aggregating around a foreign body which then becomes calcified that might be aided by the presence of turbulent air. ${ }^{11}$ Some journals stated the presence of foreign body induced chronic inflammatory reaction with deposition of mineral salts mainly calcium and magnesium. ${ }^{3}$ Orhan (2006) mentioned in his case report the rhinolith from analysis contained of calcium oxalate, calcium phosphate, magnesium and iron mineral. ${ }^{3}$ Rhinolith cases have been reported in all ages from young as 6 months up to 82 years old. ${ }^{4}$ Females are predominantly especially at third decade and rarely in children although nasal foreign bodies are common in paediatric. ${ }^{4}$ Unilateral rhinoliths are frequently seen compare to bilateral rhinoliths. ${ }^{4}$ Common routes of rhinolith are from anterior and rarely from posterior. The posterior entry into choana can be due to vomiting, sneezing or coughing. ${ }^{3}$ The rhinoliths are very often found on the nasal floor in between medial wall of maxillary sinus and inferior turbinate or between inferior turbinate with septum, midway between anterior and posterior nasal cavity. ${ }^{1,12}$ Some rare areas for example in frontal sinus, maxillary sinus, ${ }^{2}$ fossa of Rosenmuller ${ }^{13}$ and even intranasal septum also have been reported. ${ }^{14}$

Rhinoliths are accumulative in size without showing any symptoms at an initial stage. ${ }^{1}$ More often than not, asymptomatic patients were accidentally discovered rhinolith during their dental check up. ${ }^{3,16}$ Even so, long standing rhinoliths with significant enlarged can cause symptomatic to patients. Most of them complained of unilateral foul smelly nasal discharge and nasal obstruction. ${ }^{3,6}$ As in our case report, he was presented to ORL clinic for similar chief complaint. Other symptoms include local pain, foul odour, epistaxis, swelling of nose or face, anosmia, epiphora and headache. ${ }^{4}$ According to Yuca (2005), common symptoms were purulent nasal discharge, nasal obstruction and nasal malodour $(100 \%)$ then followed by headache $(57.1 \%)$, oral malodour (28.6\%) and epistaxis (14.3\%). ${ }^{17}$ The foul-smelling purulent nasal discharge is on account of the bacterial infection that precipitated from the stasis of nasal secretion. ${ }^{18}$ Clinical examinations including anterior rhinoscopy, nasoendoscopy or probing the mass can further validate the diagnosis of rhinoliths. ${ }^{14}$ They can appear as grayish, brownish, blackish or sometimes greenish mass with gritty, hard, irregular mass on probing. ${ }^{5,16}$ The endoscopy is simple, fast and non invasive procedure. Radiological assessments such as plain paranasal sinus $\mathrm{x}$-ray or dental radiograph may helpful by showing opacity lesion. However, sometime they are not visible due to superimpose adjacent structure unless can be confirmed under direct vision or endoscopic examination. ${ }^{9} \mathrm{CT}$ scan of paranasal sinus is a definitive diagnostic tool. Kocasarac (2013) in his case report mentioned that CT scans or cone beam CT are necessary before removal to identify and locate rhinoliths alongside measure the size precisely. Moreover, it also can make of differential diagnosis. ${ }^{16}$ Nevertheless, differ from Seth (2011) who stated CT scan can detect complications of rhinolith but unable to differentiate a rhinolith from other calcified mass. ${ }^{19}$ The CT scan of paranasal sinus will show homogenous high density lesion with smooth mineralization. ${ }^{3,20}$

In this reported case, the rhinolith was not visualized from the anterior rhinoscopyexamination as the mass was located in inferior meatus midway of anterior and posterior right nasal cavity. The rhinolith was diagnosed endoscopically showed dark brownish, gritty and hard mass on probing. We did not proceed further imaging procedure such as CT scan as the clinical examination was consistent with rhinolith. Simultaneously, CT scan is cost and time consuming. 
Hence, CT scan are only done under complicated case or to distinguish from other differential diagnosis. The symptoms of nasal obstruction or nasal discharge might be mistaken for other diseases such as rhinitis or sinusitis. ${ }^{21}$ They can be wrongly manage especially in primary care centre for a long period due to misdiagnosis. Despite negative history of foreign body or trauma, plain radiograph may helpful if there is at least of suspicion. Thus, referral to ORL is needed for further evaluation. Diagnosis of unilateral mass can be mistaken as there is several differential diagnosis that mimics calcified mass. They can be malignancy, benign tumour or inflammatory pathology. The malignancy includes osteosarcoma, chondrosarcoma and squamous cell carcinoma. While the benign lesion such as osteoma, calcified nasal polyps, odontoma and dermoid. Inflammatory pathology including syphilis and tuberculosis with calcification. ${ }^{3}$

The long standing of rhinoliths can cause anatomical alteration of inferior turbinate that displaced superiorly with widen the inferior meatus ${ }^{2}$ or deviated nasal septum. They may expand in nasal cavity then eroded adjacent structures which leads to palatal perforation, septal perforation or oronasal fistula. ${ }^{3}$ Moreover, they can grow and extend further to maxillary sinus and some reported to intracranial extension. ${ }^{17}$ Recurrent otitis media, vestibulitis and dacrocystitis also have been reported. ${ }^{14}$ Rhinoliths may cause obstruction of osteomeatal complex lead to sinusitis but it is rarely occur. ${ }^{17}$ Removal of rhinolith under general anaesthesia is preferable especially in children. ${ }^{4}$ Although removal of small rhinolith can be done under local anaesthesia however, it is not advisable because rhinoliths can induce massive bleeding and unbearable pain. ${ }^{9}$ There are various ways of surgical technique depending on the location and size of the rhinoliths. ${ }^{3}$ The most acceptable and less invasive is endoscopic approach. The rhinoliths are removed via anterior approach by rigid nasal endoscopy. ${ }^{17}$ It can be removed by crushing the rhinoliths into fragments or piecemeal by using appropriate forcep if the rhinoliths are larger in size. ${ }^{17}$ Irrigation and suctioning ${ }^{6}$ as well as lithotripsy ${ }^{23}$ are other alternative treatments. Regardless, if complications occur, more extensive surgery may be needed. ${ }^{12}$ For complicated cases, lateral rhinotomy can be performed. ${ }^{6}$ Some centre had done Le Fort 1 surgery for larger rhinolith and best access to remove rhinolith with repair of oroantral fistula. ${ }^{23}$ Post operative bleeding may require cauterisation or nasal packing. Antibiotic is needed when there is sign of infection. ${ }^{3}$

\section{Conclusion}

Unilateral foul smelly nasal discharge or unilateral nasal block should be suspected of rhinolith as one of the differential diagnosis in adult even though rhinoliths are rare and negative history of nasal foreign body. Diagnosis of rhinolith can be from clinical examination including nasoendoscopic procedure. CT scan is not routinely done unless it is a complex case. The recommended treatment is removal under general anaesthesia to prevent further trauma to nasal mucosa, massive epistaxis and intolerable pain.

\section{Conflict of interest: Not declared}

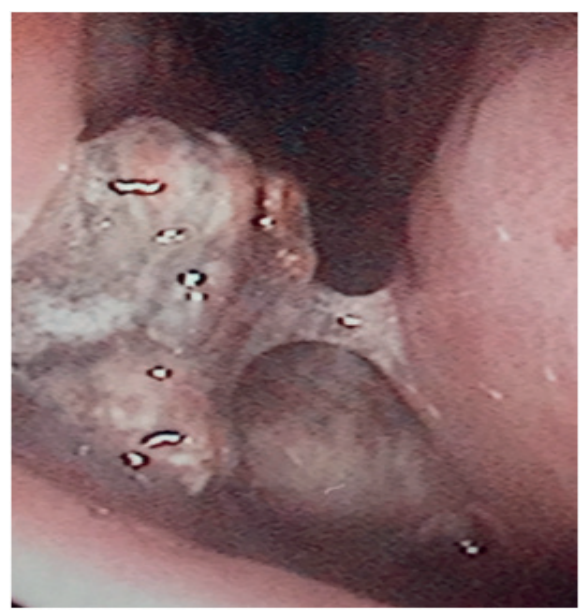

Fig. 1. Rigid nasal endoscopy shows the rhinolith in the right nasal cavity

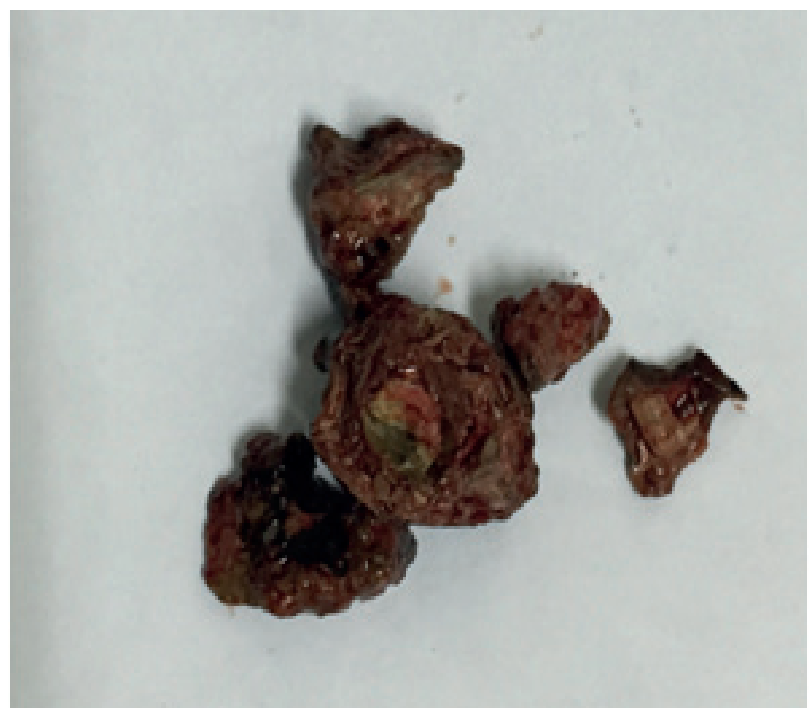

Fig. 2. Gross appearance of rhinolith fragments 


\section{$\underline{\text { References }}$}

1. Appleton SS, Kimbrough RE, Engstrom HI. Rhinolithiasis: a review. Oral Surg Oral Pathol Oral RadiolEndod. 1988; 65:693-8 https://doi.org/10.1016/0030-4220(88)90012-6

2. Chatziavramidis A et al. Longstanding rhinolith leading to anatomical alterations of ipsilateral inferior nasal meatus and turbinate. 2010; doi:10.1136/bcr.07.2010.3155 https://doi.org/10.1136/bcr.07.2010.3155

3. Orhan $\mathrm{K}$ et al. Rhinolithiasis: An uncommon entity of the nasal cavity. Oral Surg Oral Med Oral Pathol Oral RadiolEndod. 2006; 101: E28-32 https://doi.org/10.1016/j.tripleo.2005.07.036

4. LarrierD,DhingraJ.Radiologyquizcase 1.Rhinolith.Arch Otolaryngol Head Neck Surg. 2004; 130: 1340, 1342-1343 https://doi.org/10.1001/archotol.130.11.1340

5. Kalan A, Tariq M. Foreign bodies in the nasal cavities. a comprehensive review of the etiology, diagnostic pointers and therapeutic measures. Postgrad Med J. 2000;76: 484-487 https://doi.org/10.1136/pmj.76.898.484

6. Hadiet al. Rhinolithiasis: a forgotten entity. Otolaryngol Head Neck Surg. 2002; 126:486- 90 https://doi.org/10.1067/mhn.2002.121018

7. Balatsouras D et al. Rhinolithiasis: an unusual cause of nasal obstruction. Rhinology. 2002;40:162-4

8. Polson CJ.Onrhinoliths. JLaryngolOtol. 1943;58:79-116 https://doi.org/10.1017/S0022215100011002

9. Kinger A, Kawatra M. Endogenous adult rhinolith. Indian Journal of Basic and Applied Medical Research. 2014;3(2):517-520

10. Irfan $\mathrm{M}$ et al. Unilateral foul smelly nasal discharge in an adult. Malaysian Family Physician. 2012; 7(1): 28-30

11. Carney AS, Patel N, Clerke R. Foreign bodies in the ear and the aerodigestive tract in children. Scott Brown's Otorhinolarungology, Head and Neck, seventh edition. 2008:1(92):1186 https://doi.org/10.1201/b15118-105
12. Aksungur $\mathrm{EH}$ et al. A rhinolith which mimicking a nasal benign tumor. Eur J Radiol. 1999;31: 53-5 https://doi.org/10.1016/S0720-048X(98)00031-X

13. Shilston J, Foo SH, Oko M. Rhinolith in the fossa of Rosenmuller- a hidden stone. BMJ case Rep. 2011;doi: 10-1136

14. Keck $\mathrm{T}$ et al.Rhinolith of the nasal septum. International Journal of Paediatric Otorhinolaryngology. 2000; 53: $225-228$ https://doi.org/10.1016/S0165-5876(00)82011-X

15. Yaroko AA, Mohamad I, Hashim HZ. Rhinolith: An important cause of foul-smelling nasal discharge. Malaysian Family Physician. 2014; 9(1): 30-32

16. Kocasarac HD et al. Clinical and radiological aspect of rhinolith: report of five cases. Oral SurgOral Med Oral Pathol Oral Radiol. 2013; 116: 232- 237 https://doi.org/10.1016/j.oooo.2013.05.019

17. Yuca $\mathrm{K}$ et al. The importance of rigid nasal endoscopy in the diagnosis and treatment of rhinolithiasis. AurisNasus Larynx. 2006; 33: 19-22 https://doi.org/10.1016/j.anl.2005.05.013

18. Mohamad I, Arul Arumugam P. Masked rhinolith: The significance of unilateral symptom. Malaysian Family Physician. 2013; 8(1): 42-44

19. Seth S, Kumar H. Rhinolithiasis: A case report. Journal of clinical and diagnostic Research. 2011;5(4): 856-858

20. Royal SA, Gardner RE. Rhinolithiasis: an unusual pediatric nasal mass. Pediatr Radio. 1998;28: 54-5 https://doi.org/10.1007/s002470050292

21. Flood TR. Rhinolith. An unusual pediatric nasal mass. PediatrRadiol. 1966; 28(1): 54-5

22. Mink A, Gati I, Szekely J. Nasolith removal with ultrasound lithotripsy. J NHO. 1991;39:116-7

23. Bayram B. A Simple Surgical Approach for Management of Rhinoliths: Case Report. J Oral Maxillofac Surg. 2011;69:1403-14 https://doi.org/10.1016/i.joms.2010.05.072 